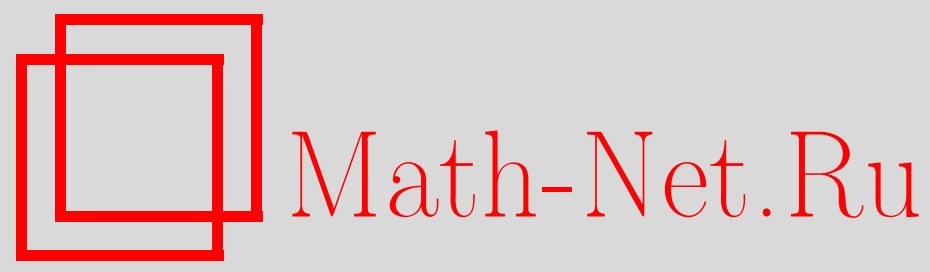

В. А. Деркач, О расширениях оператора Лагерра в пространствах с индефинитной метрикой, Матем. заметки, 1998, том 63, выпуск 4, 509-521

DOI: https://doi.org/10.4213/mzm1311

Использование Общероссийского математического портала Math-Net.Ru подразумевает, что вы прочитали и согласны с пользовательским соглашением http://www.mathnet.ru/rus/agreement

Параметры загрузки:

IP: 54.198 .67 .100

26 апреля 2023 г., 13:32:47

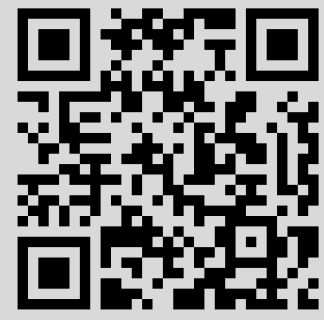




\section{О РАСШИРЕНИЯХ ОПЕРАТОРА ЛАГЕРРА \\ В ПРОСТРАНСТВАХ С ИНДЕФИНИТНОЙ МЕТРИКОЙ}

\section{В. А. Деркач}

Полиномы Лагерра-Сонина $L_{n}^{(\alpha)}$ являются ортогональными в некотором линейном пространстве с индефинитной метрикой при $\alpha<-1$. Построено пополнение П $(\alpha)$ этого пространства и дано описание самосопряженных расширений оператора Лагерра $l(y)=x y^{\prime \prime}+(1+\alpha-x) y^{\prime}, \alpha<-1$, в пространстве П $(\alpha)$. В частности, указано самосопряженное расширение оператора Лагерра, собственные функции которого совпадают с полиномами Лагерра-Сонина и образуют ортогональный базис в П $(\alpha)$.

Библиограффия: 19 названий.

1. Введение. При $\alpha>-1$ полиномы Лагерра-Сонина

$$
L_{n}^{(\alpha)}(x)=\frac{1}{n !} e^{x} x^{-\alpha}\left(e^{-x} x^{n+\alpha}\right)^{(n)}, \quad n \in \mathbb{Z}_{+},
$$

являются собственньпи функциями некоторого самосопряженного оператора $A_{\alpha}$, aсcоциированного с дифференциальным выражением $l_{\alpha}(y)=x y^{\prime \prime}+(1+\alpha-x) y^{\prime}$, и образуют ортонормированную систему в гильбертовом пространстве $L_{2}\left(\mathbb{R}_{+}, w_{\alpha}\right), w_{\alpha}=x^{\alpha} e^{-x}$. В случае $\alpha<-1$ оператор, порожденньй в $L_{2}\left(\mathbb{R}_{+}, w_{\alpha}\right)$ дифференциальным выражением $l_{\alpha}$, также является самосопряженньм (и даже унитарно эквивалентным оператору $A_{-\alpha}$ в пространстве $\left.L_{2}\left(\mathbb{R}_{+}, w_{\alpha}\right)\right)$. Однако, полиномы Лагерра-Сонина, удовлетворяющие по-прежнему уравнению $l_{\alpha}\left(L_{n}^{(\alpha)}\right)=-n L_{n}^{(\alpha)}, n \in \mathbb{Z}_{+}$, уже не принадлежат пространству $L_{2}\left(\mathbb{R}_{+}, w_{\alpha}\right)$ (при $\left.\alpha<-1\right)$.

В работе [1] было замечено, что при $-n-1<\alpha<-n$ полиномы Лагерра-Сонина $L_{n}^{(\alpha)}$ ортогональны в пространстве с индефинитной метрикой

$$
\langle y, z\rangle_{\alpha}=\int_{0}^{\infty} x^{\alpha}\left(e^{-x} y \bar{z}-\sum_{j=0}^{n-1}\left(e^{-x} y \bar{z}\right)^{(j)}(0) \frac{x^{j}}{j !}\right) d x
$$

В связи с этим было бы естественно построить самосопряженньй оператор, порожденный дифференциальным выражением $l_{\alpha}$, не в $L_{2}\left(\mathbb{R}_{+}, w_{\alpha}\right)$, а в пространстве Понтрягина с индефинитной метрикой (1). Эта задача была рассмотрена в [2], [3], для полиномов типа Якоби - в [4]. Заметим однако, что пополнение пространства с метрикой (1) в [2] было описано неверно.

Работа выполнена при частичной поддержке фонда INTAS, грант № 93-02449. 
Аналогичная задача возникает в теории обобщенных точечных взаимодействий, где для устранения расходимостей вводится перенормированная метрика, похожая на метрику (1). Так, Ф.А. Березин построил в [5] теорию расширений эрмитовых операторов в пространстве с такой метрикой на алгебраическом уровне, не рассматривая пополнения этого пространства. Описание замыкания такого пространства и действующих в нем операторов получено в [6]. В [7] рассмотрены общие модели самосопряженных операторов, действующих в пространствах с индефинитной метрикой (1).

В настоящей работе мы применяем технику, развитую в [7], к описанию замыкания $\Pi(\alpha)$ пространства с индефинитной метрикой (1) и показьваем, что минимальньй оператор $A$ в $\Pi(\alpha)$, порожденный выражением $l_{\alpha}$, является симметричным с индексами дефекта 1. Интересно заметить, что оператор $A$ оказывается неплотно заданным. Построены граничные операторы и в их терминах описаны самосопряженные расширения оператора $A$. В частности, указано самосопряженное расширение оператора $A$, для которого полиномы Лагерра-Сонина являются собственными функциями.

2. Предварительные сведения. Напомним некоторые понятия теории расширений эрмитовых операторов из [8]-[12]. Пусть $A$ - эрмитов оператор в пространстве Понтрягина $\left(\Pi_{\varkappa},[\cdot, \cdot]\right), \varkappa \in \mathbb{Z}_{+}$, с неплотной, вообще говоря, областью определения $\mathscr{D}(A)$. В дальнейшем оператор $A$ отождествляется с его графиком $\{\{y, A y\}$ : $y \in \mathscr{D}(A)\}$, так что множество замкнутых линейных операторов оказывается вложенным в множество замкнутых линейных отношений, т.е. подпространств в $\Pi_{\varkappa}$ (см. [9]). Определим сопряженное линейное отношение $A^{+}$в $\Pi_{\varkappa}$, полагая

$$
A^{+}=\left\{\{z, \dot{z}\} \in \Pi_{\varkappa} \oplus \Pi_{\varkappa}:[\dot{z}, y]=[z, \dot{y}] \forall\{y, \dot{y}\} \in A\right\} \subset \Pi_{\varkappa} \oplus \Pi_{\varkappa}
$$

Многозначная часть mul $A^{+}:=\left\{\dot{z} \in \Pi_{\varkappa}:\{0, \dot{z}\} \in A^{+}\right\}=\Pi_{\varkappa}[-] \mathscr{D}(A)$ отношения $A^{+}$ отлична от 0, если $\overline{\mathscr{D}(A)} \neq \Pi_{\varkappa}$. Положим $\mathscr{N}_{\infty}=\Pi_{\varkappa}[-] \mathscr{D}(A), \mathscr{N}_{\lambda}=\Pi_{\varkappa}[-](A-\bar{\lambda}) \mathscr{D}(A)$, $\lambda \in \mathbb{C}$. Дефектные подпространства $\mathcal{N}_{\lambda}$ имеют одинаковую размерность $n_{ \pm}$для всех $\lambda \in \mathbb{C}_{ \pm}$за исключением, быть может, конечного числа точек.

ОПРЕДЕЛЕНИЕ 1. Совокупность $\left\{\mathscr{H}, \Gamma_{0}, \Gamma_{1}\right\}$, в которой $\mathscr{H}$ - гильбертово пространство, а $\Gamma_{0}, \Gamma_{1}$ - ограниченные линейные операторы из $A^{+}$в $\mathscr{H}$, назьвают граничной тройкой отношения $A^{+}[13]$, если

1) отображение $\Gamma: \widehat{z} \rightarrow\left\{\Gamma_{0} \widehat{z}, \Gamma_{1} \widehat{z}\right\}$ из $A^{+}$в $\mathscr{H} \oplus \mathscr{H}$ сюръективно;

2) для всех $\widehat{y}=\{y, \dot{y}\}, \widehat{z}=\{z, \dot{z}\} \in A^{+}$справедливо равенство

$$
[\dot{y}, z]-[y, \dot{z}]=\left(\Gamma_{1} \widehat{y}, \Gamma_{0} \widehat{z}\right) \mathscr{H}-\left(\Gamma_{0} \widehat{y}, \Gamma_{1} \widehat{z}\right) \mathscr{H}
$$

Легко видеть (см. [11]), что эрмитов оператор $A$ в $\left(\Pi_{\varkappa},[\cdot, \cdot]\right)$ с равнњми дефектньми числами $n_{+}=n_{-}$обладает граничной тройкой. В дальнейшем мы будем считать, что $n_{+}=n_{-}=1$. Тогда $\mathscr{H}=\underset{\widetilde{C}}{\mathbb{C}} \mathrm{C}$ каждой граничной тройкой естественно связано самосопряженное расширение $\widetilde{A}_{\infty}=\operatorname{ker} \Gamma_{0}$ оператора $A$. Всякое его самосопряженное расширение $\widetilde{A}$, отличное от $\widetilde{A}_{\infty}$, имеет вид

$$
\widetilde{A}_{\theta}=\operatorname{ker}\left(\Gamma_{1}-\theta \Gamma_{0}\right), \quad \theta \in \mathbb{R}
$$


ОПРЕДЕЛЕНИЕ 2 . Функция $m(\lambda)$, определенная равенством

$$
m(\lambda)=\frac{\Gamma_{1} \widehat{y}_{\lambda}}{\Gamma_{0} \widehat{y}_{\lambda}}, \quad \widehat{y}_{\lambda}=\left\{y_{\lambda}, \lambda y_{\lambda}\right\}, \quad y_{\lambda} \in \mathscr{N}_{\lambda}, \quad \lambda \in \rho\left(\widetilde{A}_{\infty}\right),
$$

назьвается функиией Вейля оператора $A$, отвечающей граничной тройке $\left\{\mathbb{C}, \Gamma_{0}, \Gamma_{1}\right\}$.

$\Phi$ ункция Вейля $m(\lambda)$ простого эрмитова оператора $A$ в пространстве Понтрягина $\Pi_{\varkappa}$ является его $Q$-функцией (в смысле определения из [14]) и принадлежит обобщенному классу Неванлинны $N_{\varkappa}$, т.е. ядро

$$
\frac{m(\lambda)-m(\bar{\zeta})}{\lambda-\bar{\zeta}}, \quad \lambda, \zeta \in \rho\left(\widetilde{A}_{\infty}\right)
$$

имеет $\varkappa$ отрищательньх квадратов в $\rho\left(\widetilde{A}_{\infty}\right)($ см. [14]).

В терминах функции Вейля может быть описан точечный спектр $\sigma_{p}\left(\widetilde{A}_{\theta}\right)$ расширения $\widetilde{A}_{\theta}$. Если $\lambda \in \rho\left(\widetilde{A}_{\infty}\right)$, то справедлива эквивалентность

$$
\lambda \in \sigma_{p}\left(\widetilde{A}_{\theta}\right) \Longleftrightarrow m(\lambda)-\theta=0 .
$$

Применим приведенную схему к рассмотрению расширений минимального дифференциального оператора $A_{\alpha}$, порожденного в $L_{2}\left(\mathbb{R}_{+}, w_{\alpha}\right)$ дифференциальным выражением $l_{\alpha}(y)=x y^{\prime \prime}+(1+\alpha-x) y^{\prime}$, в классическом случае $\alpha>-1$. При этом оператор $A_{\alpha}$ оказьвается эрмитовьм в гильбертовом пространстве $L_{2}\left(\mathbb{R}_{+}, w_{\alpha}\right)$.

Найдем дефектные подпространства оператора $A_{\alpha}$. Общее решение уравнения Куммера

$$
l_{\alpha} y-\lambda y=x y^{\prime \prime}+(1+\alpha-x) y^{\prime}-\lambda y=0
$$

имеет вид (см. [15])

$$
y=C_{1} \Phi(\lambda, 1+\alpha, x)+C_{2} \Psi(\lambda, 1+\alpha, x),
$$

где $\Phi(\lambda, 1+\alpha, x), \Psi(\lambda, 1+\alpha, x)$ - вырожденные гипергеометрические функции. Асимптотика функций $\Phi(\lambda, 1+\alpha, x), \Psi(\lambda, 1+\alpha, x)$ при $x \rightarrow+\infty$ (см. [15])

$$
\begin{aligned}
& \Phi(\lambda, 1+\alpha, x)=\frac{\Gamma(1+\alpha)}{\Gamma(\lambda)} e^{x} x^{\lambda-(1+\alpha)}\left(1+O\left(\frac{1}{x}\right)\right) \\
& \Psi(\lambda, 1+\alpha, x)=x^{-\lambda}\left(1+O\left(\frac{1}{x}\right)\right)
\end{aligned}
$$

показывает, что для всех $\alpha>-1$ имеет место случай предельной точки в $\infty$. Учитьвая поведение функций $\Phi(\lambda, 1+\alpha, x), \Psi(\lambda, 1+\alpha, x)$ при $x \rightarrow 0$ (см. [15]): $\Phi(\lambda, 1+\alpha, x) \asymp 1$,

$$
\Psi(\lambda, 1+\alpha, x) \asymp \begin{cases}x^{-\alpha} \Gamma(\alpha) / \Gamma(\lambda), & \alpha \in(0, \infty), \\ -\ln x / \Gamma(\lambda), & \alpha=0, \\ \Gamma(-\alpha) / \Gamma(\lambda-\alpha), & \alpha \in(-1,0),\end{cases}
$$

получим, что при всех $\alpha \geqslant 1$ имеет место случай предельной точки в 0, а при $|\alpha|<1-$ случай предельного круга в 0 . Таким образом, при $\alpha \geqslant 1$ минимальньй оператор $A_{\alpha}$ является самосопряженным в $L_{2}\left(\mathbb{R}_{+}, w_{\alpha}\right)$. Если же $|\alpha|<1$, то оператор $A_{\alpha}$ симметрический, его индексы дефекта равны 1 , а дефектное подпространство $\mathscr{N}_{\lambda}$ порождено функцией $\Psi(\lambda, 1+\alpha, x)$. 
Зафиксируем $\lambda \in \mathbb{C}_{+}$и рассмотрим линеал $\mathscr{D}=C_{0}^{2}\left(\mathbb{R}_{+}\right)+\mathscr{N}_{\lambda}+\mathscr{N}_{\bar{\lambda}}$. При $\alpha \in(-1,0)$ для всех $y, z \in \mathscr{D}$ справедливо равенство

$$
\begin{aligned}
\left(l_{\alpha} y, z\right)-\left(y, l_{\alpha} z\right) & =\int_{0}^{\infty} x^{\alpha} e^{-x}\left(l_{\alpha}(y) \bar{z}-y l_{\alpha}(\bar{z})\right) d x=\int_{0}^{\infty}\left(x^{\alpha+1} e^{-x} W(\bar{z}, y)\right)^{\prime} d x \\
& =B_{1} y \overline{B_{0} z}-\Gamma_{0} y \overline{\Gamma_{1} z}
\end{aligned}
$$

в котором $W(\bar{z}, y)=y^{\prime} \bar{z}-y \bar{z}^{\prime}$, а операторы $\Gamma_{i}: \mathscr{D} \rightarrow \mathbb{C}, i=0,1$, определены соотношениями

$$
\Gamma_{0} y=\lim _{x \downarrow 0} x^{\alpha+1} y^{\prime}(x), \quad \Gamma_{1} y=\lim _{x \downarrow 0} y(x), \quad-1<\alpha<0 .
$$

Из равенства [15, с. 324]

$$
\Psi^{\prime}(\lambda, 1+\alpha, x)=-\lambda \Psi(\lambda+1,2+\alpha, x) \asymp-\lambda \frac{\Gamma(1+\alpha)}{\Gamma(1+\lambda)} x^{-(1+\alpha)}, \quad \alpha>-1,
$$

следует, что выражение для $\Gamma_{0} y$ в (4) корректно определено, если $y \in \mathscr{D}$. Корректность определения (4) для $\Gamma_{1} y$ следует из (2).

В силу равенства $\left(\Gamma_{1} \oplus \Gamma_{0}\right)\left(\mathcal{N}_{\lambda}+\mathscr{N}_{\bar{\lambda}}\right)=\mathbb{C} \oplus \mathbb{C}$ из формулы $(3)$ следует, что операторы $\Gamma_{1}, \Gamma_{0}$ допускают непрерывное продолжение до ограниченных операторов, действующих из $\mathscr{D}\left(A_{\alpha}^{*}\right)$ с нормой графика оператора $A_{\alpha}^{*}$ в $\mathbb{C}$. При этом формула $(3)$, являющаяся аналогом тождества Лагранжа, остается справедливой для всех $y, z \in \mathscr{D}\left(A_{\alpha}^{*}\right)$. Таким образом, совокупность $\left\{\mathbb{C}, \Gamma_{0}, \Gamma_{1}\right\}$ образует граничную тройку оператора $A_{0}^{*}$.

Вычислим соответствующую функцию Вейля. В случае $-1<\alpha<0$ из (2), (4), (5) получаем

$$
\Gamma_{0} \Psi(\lambda)=-\lambda \frac{\Gamma(1+\alpha)}{\Gamma(1+\lambda)}=-\frac{\Gamma(1+\alpha)}{\Gamma(\lambda)}, \quad \Gamma_{1} \Psi(\lambda)=\frac{\Gamma(-\alpha)}{\Gamma(\lambda-\alpha)} .
$$

Согласно определению 2 функция Вейля $m_{\alpha}(\lambda)$ имеет вид

$$
m_{\alpha}(\lambda)=-\frac{\Gamma(\lambda) \Gamma(-\alpha)}{\Gamma(\lambda-\alpha) \Gamma(1+\alpha)}=-\frac{B(\lambda,-\alpha)}{\Gamma(1+\alpha)}, \quad-1<\alpha<0,
$$

где $B(\lambda,-\alpha)$ - бэта-функция Эйлера.

Аналогично, в случае $0 \leqslant \alpha<1$ граничный оператор $\Gamma_{0}$ определим равенством (4), а для $\Gamma_{1} y$ положим

$$
\begin{gathered}
\Gamma_{1} y=\lim _{x \downarrow 0}\left(y(x)+\frac{x}{\alpha} y^{\prime}(x)\right), \quad 0<\alpha<1, \\
\Gamma_{1} y=\lim _{x \downarrow 0}\left(y(x)-x y^{\prime}(x)(2 \gamma+\ln x)\right), \quad \alpha=0 .
\end{gathered}
$$

Здесь $\gamma$-постоянная Эйлера. Соответствующая функция Вейля $m_{\alpha}(\lambda)$ выгисляется по формуле (6) при $\alpha \in(0,1)$ и совпадает с $\psi$-функцией при $\alpha=0$ :

$$
m_{0}(\lambda)=\psi(\lambda):=\frac{d}{d \lambda} \Gamma(\lambda) .
$$

Заметим, что функция Вейля $m_{\alpha}(\lambda)$ допускает разложение [16, гл. 12])

$$
m_{\alpha}(\lambda)=-\frac{1}{\alpha \Gamma(\alpha+1)} \sum_{k=0}^{\infty} \frac{(\alpha+1)_{k}}{k !(\lambda+k)}
$$

в котором $(\alpha+1)_{k}=(\alpha+1) \cdots(\alpha+k),(\alpha+1)_{0}=1$. Из (9) следует, что $m_{\alpha}(\lambda)$ является функцией класса Неванлинны $N:=N_{0}$, т.е. $m_{\alpha}(\lambda)$ голоморфна в $\mathbb{C}_{+} \cup \mathbb{C}_{-}$, симметрична $\left(m_{\alpha}(\bar{\lambda})=\overline{m_{\alpha}(\lambda)}\right)$ и имеет в $\mathbb{C}_{+}$неотрищательную мнимую часть. Суммируя сказанное, получим следующее 
ПРЕДЛОЖЕНИЕ 1. При $\alpha \in(-1,1)$ минимальный оператор $A_{\alpha}$ является симметрическим оператором в $L_{2}\left(\mathbb{R}_{+}, w_{\alpha}\right)$ с индексами дефекта $n_{+}=n_{-}=1$, дефектное подпространство $\mathcal{N}_{\lambda}$ порождено функцией $\Psi(\lambda, 1+\alpha, x)$. При этом

1) граничная тройка оператора $A_{\alpha}^{*}$ может быть определена соотношениями (4) при $\alpha \in(0,1)$ u (7) прu $\alpha \in(-1,0]$;

2) соответствующая функиия Вейля $m_{\alpha}(\lambda)$ вычисляется по формулам (6) при $\alpha \neq 0 u(8)$ nрu $\alpha=0$;

3) всякое самосопряженное расширение $\widetilde{A}_{\alpha, \theta}$ оператора $A_{\alpha}$ характеризуется условиями $у \in \mathscr{D}\left(\widetilde{A}_{\alpha, \theta}\right) \Longleftrightarrow y \in \mathscr{D}\left(A_{\alpha}^{*}\right)$,

$$
\Gamma_{1} y=\theta \Gamma_{0} y, \quad \theta \in \overline{\mathbb{R}}
$$

при $\theta=\infty$ граничное условие (10) приобретает вид

$$
\Gamma_{0} y=\lim _{x \downarrow 0} x^{\alpha+1} y^{\prime}(x)=0
$$

4) спектр расширения $\widetilde{A}_{\alpha, \theta}$ coстоит из простых собственных чисел и при $\theta \in \mathbb{R}$ совпадает с нулями функиии $m_{\alpha}(\lambda)-\theta$.

Отметим, что именно расширение $\widetilde{A}_{\alpha, \infty}$ имеет собственные функции, совпадающие с полиномами Лагерра-Сонина $L_{n}^{(\alpha)}(x)$.

3. Пространства Лагерра. Для того чтобы перейти к случаю $\alpha<-1$, нам потребуется следующая лемма, доказанная в [7].

Лемма 1. Пусть Я - линейное пространство, снабженное скалярным произведением $(\cdot, \cdot)$, и $v_{1}, \ldots, v_{n}$ - система линейных функиионалов, заданная на Я્, такая, что ни одна из их линейных комбинаций не является непрерывной в полунорме $(\cdot, \cdot)^{1 / 2}$. Далее, пусть $G=\left(g_{j k}\right)_{j, k=1}^{n}-$ невырохсденная эрмитова $(n \times n)$-матрица, имеющая $\varkappa$ отрицательных $(n-\varkappa$ положсительных) собственных значений, и в $\mathscr{P}$ задано внутреннее произведение

$$
\langle y, z\rangle=(y, z)+\sum_{j, k=1}^{n} g_{j k} v_{k}(y) \overline{v_{j}(z)}
$$

Тогда это внутреннее произведение имеет $\varkappa$ отрииательных квадратов на Я્Р. Пополнение пространства Я્P по метрике (11) есть пространство Понтрягина $\mathscr{H} \oplus \mathscr{G}$, где $\mathscr{H}$ - пополнение факторпространства $\mathscr{P} / \mathscr{P}^{0}, \mathscr{P}^{0}=\{y \in \mathscr{P}:(y, y)=0\}$ $u \mathscr{G}=\left(\mathbb{C}^{n},(G \cdot, \cdot) \mathbb{C}^{n}\right)$.

Пусть $n \in \mathbb{N},-n-1<\alpha<-n, \mathscr{P}$ - множество полиномов с индефинитной метрикой (1). Положим для $y(x) \in C^{\infty}\left(\mathbb{R}_{+}\right)$

$$
y^{\{k\}}(x)=y(x)-\sum_{j=0}^{k-1} y^{(j)}(0) \frac{x^{j}}{j !}
$$


и построим отображение $i_{\alpha}$ из $\mathscr{P}$ в $L_{2}\left(\mathbb{R}_{+}, w_{\alpha+2 \varkappa}\right) \oplus \mathbb{C}^{2 \varkappa}, \varkappa=[(n+1) / 2]$ :

$$
i_{\alpha}: y \rightarrow\left(\frac{y^{\{\varkappa\}}(x)}{x^{\varkappa}}, y_{0}, \ldots, y_{\varkappa-1}, Y_{\varkappa-1}, \ldots, Y_{0}\right) \text {, }
$$

где

$$
\begin{gathered}
Y_{j}=\int_{0}^{\infty} x^{\alpha+j} e^{-x} y^{\{n-j\}}(x) d x+\sum_{i=\varkappa}^{n-j-1} \frac{1}{i !} y^{(i)}(0) \Gamma(\alpha+i+j+1), \\
y_{j}=\frac{1}{j !} y^{(j)}(0), \quad 0 \leqslant j \leqslant \varkappa-1 .
\end{gathered}
$$

Следующая теорема описьвает замыкание П $(\alpha)$ линеала с метрикой $(1)$, называемого в [2] пространством Лагерра. Ее доказательство весьма близко к доказательству соответствующей теоремы в [7] (с тем лиш отличием, что $n$ может быть нечетным).

Теорема 1. Пусть $-n-1<\alpha<-n, n \in \mathbb{N}$. Тогда пополнение П $(\alpha)$ пространства $\left(C^{\infty}\left(\mathbb{R}_{+}\right),\langle\cdot, \cdot\rangle_{\alpha}\right)$ является пространством Понтрягина с индексом $\varkappa=[(n+1) / 2]$. Линейное отображкение $i_{\alpha}$ является изометрическим изоморфизмом межсду $(\alpha)$ и $\pi_{\varkappa}-$ пространством $L_{2}\left(\mathbb{R}_{+}, w_{\alpha+2 \varkappa}\right) \oplus\left(\mathbb{C}^{2 \varkappa},\left(G_{\alpha} \cdot, \cdot\right)\right), \boldsymbol{в}$ котором оператор Грамма $G_{\alpha}$ задается матричей

$$
G_{\alpha}=\left(\begin{array}{ccc|ccc}
\Gamma(\alpha+1) & \ldots & \Gamma(\alpha+\varkappa) & \mathbf{0} & & 1 \\
\vdots & . \cdot & \vdots & & . \cdot & \\
\Gamma(\alpha+\varkappa) & \ldots & \Gamma(\alpha+2 \varkappa-1) & 1 & & \mathbf{0} \\
\hline 0 & \ldots & 1 & & 0 & \\
1 & & & 0 & &
\end{array}\right) .
$$

ДокАЗАТЕЛЬСТво. Воспользуемся тождеством

$$
(f g)^{\{n\}}(x)=f(x) g^{\{n\}}(x)+\sum_{j=0}^{n-1} g^{(j)}(0) f^{\{n-j\}}(x) \frac{x^{j}}{j !}, \quad f, g \in C^{\infty}\left(\mathbb{R}_{+}\right)
$$

Полагая в (13) $f(x)=e^{-x}, g(x)=y(x) \bar{z}(x)$ и учитывая соотношения

$$
\int_{0}^{\infty} x^{\alpha+j}\left(e^{-x}\right)^{\{n-j\}} d x=\Gamma(\alpha+j+1), \quad j=0, \ldots, n-1,
$$

представим форму $\langle y, z\rangle_{\alpha}$ в виде

$$
\langle y, z\rangle_{\alpha}=\int_{0}^{\infty} x^{\alpha} e^{-x}(y \bar{z})^{\{n\}}(x) d x+\sum_{j=0}^{n-1} \frac{1}{j !}(y \bar{z})^{(j)}(0) \Gamma(\alpha+j+1) .
$$

Далее воспользуемся соотношением (см. [7])

$$
(y \bar{z})^{\{n\}}(x)=y^{\{\varkappa\}} \bar{z}^{\{\varkappa\}}+\sum_{j=0}^{\varkappa-1} x^{j}\left(y_{j} \bar{z}^{\{n-j\}}+y^{\{n-j\}} \bar{z}_{j}\right), \quad n \in \mathbb{N} .
$$


Подставляя (15) в (14) и учитьвая равенство (12), получим

$$
\begin{aligned}
\langle y, z\rangle_{\alpha}= & \int_{0}^{\infty} x^{\alpha} e^{-x} y^{\{\varkappa\}} \bar{z}^{\{\varkappa\}} d x+\sum_{j=0}^{n-1} \sum_{i=0}^{j} y_{j-i} \bar{z}_{i} \Gamma(\alpha+j+1) \\
& +\sum_{j=0}^{\varkappa-1}\left(y_{j} \int_{0}^{\infty} x^{\alpha+j} e^{-x} \bar{z}^{\{n-j\}} d x+\bar{z}_{j} \int_{0}^{\infty} x^{\alpha+j} e^{-x} y^{\{n-j\}} d x\right) \\
= & \int_{0}^{\infty} x^{\alpha} e^{-x} y^{\{\varkappa\}} \bar{z}^{\{\varkappa\}} d x+\sum_{j=0}^{\varkappa-1} \sum_{i=0}^{j} y_{j-i} \bar{z}_{i} \Gamma(\alpha+j+1)+\sum_{j=0}^{\varkappa-1}\left(y_{j} \bar{Z}_{j}+Y_{j} \bar{z}_{j}\right),
\end{aligned}
$$

где $z_{j}, Z_{j}$ - координаты вектора $i_{\alpha}(z)=\left(z^{\{\varkappa\}}(x) / x^{\varkappa}, z_{0}, \ldots, z_{\varkappa-1}, Z_{\varkappa-1}, \ldots, Z_{0}\right)$.

Введем в рассмотрение полунорму

$$
(y, y)^{1 / 2}=\left(\int_{0}^{\infty} x^{\alpha+2 \varkappa} e^{-x}\left|\frac{y^{\{\varkappa\}}(x)}{x^{\varkappa}}\right|^{2} d x\right)^{1 / 2}, \quad y \in \mathscr{P} .
$$

Для того чтобы применить лемму 1 , достаточно проверить, что ни одна из линейных комбинаций функционалов

$$
v_{j}(y)= \begin{cases}y_{j}, & 0 \leqslant j \leqslant \varkappa-1, \\ Y_{2 \varkappa-j-1}, & \varkappa \leqslant j \leqslant 2 \varkappa-1,\end{cases}
$$

не является непрерьвным функционалом по отношению к полунорме (16).

Допустим это не так, т.е. существуют $c_{j}, d_{j} \in \mathbb{C}, j=0, \ldots, \varkappa-1$, и $h(x) \in L_{2}\left(w_{\alpha+2 \varkappa}\right)$ такие, что

$$
\sum_{j=0}^{\varkappa-1} c_{j} y_{j}+\sum_{j=0}^{\varkappa-1} d_{j} Y_{j}=\int_{0}^{\infty} x^{\alpha+2 \varkappa} e^{-x} \frac{y^{\{\varkappa\}}}{x^{\varkappa}} h(x) d x .
$$

Полагая в (17) $y=x^{j}$, получим $c_{j}=0$.

Пусть теперь $y \in x^{2 \varkappa-1} \mathscr{P}$. Тогда $y^{\{n-j\}}=y, j=0, \ldots, \varkappa$, и $y^{(i)}(0)=0, i=0, \ldots$, $2 \varkappa-1$. Поэтому равенство (17) принимает вид

$$
\sum_{j=0}^{\varkappa-1} d_{j} \int_{0}^{\infty} x^{\alpha+2 \varkappa} e^{-x} \frac{y}{x^{\varkappa}} x^{j-\varkappa} d x=\int_{0}^{\infty} x^{\alpha+2 \varkappa} e^{-x} \frac{y}{x^{\varkappa}} h(x) d x .
$$

Так как функции вида $y(x) / x^{\varkappa} \in x^{2 \varkappa-1} \mathscr{P}$ плотны в $L_{2}\left(w_{\alpha+2 \varkappa}\right)$, из (18) получаем

$$
\sum_{j=0}^{\varkappa-1} d_{j} x^{j-\varkappa}=h(x) \in L_{2}\left(w_{\alpha+2 \varkappa}\right)
$$

что возможно лишш при $d_{j}=0, j=0, \ldots, \varkappa-1$.

Отметим [2], что в случае $-n-1<\alpha<-n, n \in \mathbb{N}$, скалярное произведение (1) можно переписать в виде

$$
\langle y, z\rangle_{\alpha}=\gamma_{n} \int_{0}^{\infty} x^{\alpha+n}\left(e^{-x} y \bar{z}\right)^{(n)} d x, \quad \gamma_{n}=\frac{(-1)^{n} \Gamma(\alpha+1)}{\Gamma(\alpha+n+1)}>0 .
$$


4. Оператор Лагерра. Неклассический случай. Пусть $-n-1<\alpha<-n$, $n \in \mathbb{N}$. Множество $C^{\infty}\left(\mathbb{R}_{+}\right) \cap \Pi(\alpha)$ плотно в $\Pi(\alpha)$, так как оно содержит $\mathscr{P}$. Рассмотрим дифференциальное выражение $l_{\alpha}$ на множестве

$$
\mathscr{D}=\text { l.s. }\left\{C^{\infty}\left(\mathbb{R}_{+}\right) \cap \Pi(\alpha), \Psi(\lambda, 1+\alpha, x)\right\}, \quad \lambda \in \mathbb{C} \backslash \mathbb{R} .
$$

Так как для всех $y(x) \in \mathscr{D}$ существуют производные

$$
y^{(j)}(0)=\lim _{x \downarrow 0} y^{(j)}(x), \quad j=1, \ldots, n,
$$

и определены граничные операторы

$$
\Gamma_{0} y=\gamma_{n} \lim _{x \downarrow 0} x^{\alpha+n+1} y^{(n+1)}(x), \quad \Gamma_{1} y=\lim _{x \downarrow 0} y(x),
$$

из тождества (см. [3])

$$
x^{\alpha+n}\left(e^{-x}\left(\bar{z} l_{\alpha} y-y l_{\alpha} \bar{z}\right)\right)^{n}=-\left(x^{\alpha+n+1}\left(e^{-x} W(y, \bar{z})\right)^{(n)}\right)^{\prime}
$$

и равенства (19) получим

$$
\left\langle l_{\alpha} y, z\right\rangle_{\alpha}-\left\langle y, l_{\alpha} z\right\rangle_{\alpha}=-\gamma_{n} \lim _{x \downarrow 0} x^{\alpha+n+1}\left(e^{-x} W(y \bar{z})\right)^{(n)} .
$$

Отсюда следует, что для всех $y, z \in \mathscr{D}$ справедливо равенство

$$
\left\langle l_{\alpha} y, z\right\rangle_{\alpha}-\left\langle y, l_{\alpha} z\right\rangle_{\alpha}=\Gamma_{1} y \overline{\Gamma_{0} z}-\Gamma_{0} y \overline{\Gamma_{1} z} .
$$

Пусть $T_{\alpha}$ - замыкание в $\Pi(\alpha) \oplus \Pi(\alpha)$ графика оператора $l_{\alpha}$, первоначально определенного на $\mathscr{D}$. Назовем $T_{\alpha}$ максимальным отношением. Ниже будет показано, что $T_{\alpha}$ не зависит от выбора $\lambda \in \mathbb{C} \backslash \mathbb{R}$, а его многозначная часть $T_{\alpha}(0)$ отлична от 0 , если $\alpha<-1$. Так как $\left(\Gamma_{0} \oplus \Gamma_{1}\right) \mathscr{D}=\mathbb{C} \oplus \mathbb{C}$, операторы $\Gamma_{0}, \Gamma_{1}$ допускают продолжение по непрерывности до ограниченных операторов из $T_{\alpha}$ в $\mathbb{C}$. Определим также минимальный оператор $A_{\alpha}$ как замыкание в $\Pi(\alpha)$ оператора $l_{\alpha}$ с областью определения $\mathscr{D}_{0}=\{y \in \mathscr{D}: y(0)=0\}$. Отметим, что оператор $A_{\alpha}$ имеет неплотную область определения, так как вектор

$$
h=(\underbrace{0, \ldots, 0}_{\varkappa}, 1) \in \Pi(\alpha)
$$

ортогонален к $\mathscr{D}_{0}$. Таким образом, $A_{\alpha}^{+}$- замкнутое линейное отношение с ненулевой многозначной частью (см. [9]). Ясно, что $A_{\alpha} \subset T_{\alpha}$. Из (21) следует, что $T_{\alpha} \subset A_{\alpha}^{+}$и $\operatorname{dim} T_{\alpha}\left(\bmod A_{\alpha}\right)=2$.

Рассмотрим эрмитово расширение $\widetilde{A}_{\alpha, 0}=\left\{\{y, \dot{y}\} \in T_{\alpha}: B_{1}\{y, \dot{y}\}=0\right\}$ оператора $A_{\alpha}$. Покажем, что $\lambda \in \rho\left(\widetilde{A}_{\alpha, 0}\right)$. Будем искать решение уравнения

$$
x y^{\prime \prime}+(1+\alpha-x) y^{\prime}-\lambda y=f(x), \quad f \in C^{\infty}\left(\mathbb{R}_{+}\right) \cap \Pi(\alpha),
$$

удовлетворяющее условию $y(0)=0$, в виде

$$
y=C_{1}(x) \Phi_{1}(\lambda, 1+\alpha, x)+C_{2}(x) \Psi(\lambda, 1+\alpha, x),
$$


где

$$
\begin{aligned}
\Phi_{1}(\lambda, 1+\alpha, x) & =x^{-\alpha} \Phi(\lambda-\alpha, 1-\alpha, x) \\
& =\Gamma(\lambda) \Gamma(1-\alpha)\left(\frac{\Phi(\lambda, 1+\alpha, x)}{\Gamma(1+\alpha) \Gamma(\lambda-\alpha)}+\frac{\sin \pi \alpha}{\pi} \Psi(\lambda, 1+\alpha, x)\right),
\end{aligned}
$$

a $C_{1}(x), C_{2}(x)$ удовлетворяют системе уравнений

$$
C_{1}^{\prime} \Phi_{1}+C_{2}^{\prime} \Psi=0, \quad C_{1}^{\prime} \Phi_{1}^{\prime}+C_{2}^{\prime} \Psi^{\prime}=\frac{f(x)}{x} .
$$

Так как

$$
W\left(\Phi_{1}, \Psi\right)=-\frac{\Gamma(1-\alpha)}{\Gamma(\lambda-\alpha)} x^{-(1+\alpha)} e^{x}
$$

(см. $[15$, c. 322$])$, то

$$
C_{1}^{\prime}=\frac{\Gamma(\lambda-\alpha)}{\Gamma(1-\alpha)} f(x) \Psi(x) x^{\alpha} e^{-x}, \quad C_{2}^{\prime}=-\frac{\Gamma(\lambda-\alpha)}{\Gamma(1-\alpha)} f(x) \Phi_{1}(x) x^{\alpha} e^{-x} .
$$

Из (22), (23) следует

$$
\begin{aligned}
\frac{\Gamma(1-\alpha)}{\Gamma(\lambda-\alpha)} y(x)= & -\int_{x}^{\infty} f(t) \Psi(t) w_{\alpha}(t) d t \Phi(x)-\int_{0}^{x} f(t) \Phi_{1}(t) w_{\alpha}(t) d t \Psi(x) \\
= & -\int_{x}^{\infty} t^{\alpha}\left(e^{-t} f(t) \Psi(t)\right)^{\{1\}} d t \Phi(x) \\
& -\int_{0}^{x} f(t) \Phi_{1}(t) w_{\alpha}(t) d t \Psi(x)+\frac{x^{\alpha+1}}{\alpha+1} f(0) \Psi(0) \Phi(x) .
\end{aligned}
$$

Так как

$$
\Phi_{1}(x) \asymp x^{-\alpha}, \quad \Psi(x) \asymp \frac{\Gamma(-\alpha)}{\Gamma(\lambda-\alpha)} \quad \text { при } x \rightarrow 0,
$$

имеем $y(0)=0$. Таким образом, справедливо представление

$$
y(x)=\left(\widetilde{A}_{\alpha, 0}-\lambda\right)^{-1} f=-\frac{\Gamma(\lambda-\alpha)}{\Gamma(1-\alpha)} \int_{0}^{\infty} K(x, t, \lambda) f(t) w_{\alpha}(t) d t,
$$

в котором ядро $K(x, t, \lambda)$ имеет вид

$$
K(x, t, \lambda)=\left\{\begin{array}{l}
\Phi_{1}(\lambda, 1+\alpha, t) \Psi(\lambda, 1+\alpha, x), \quad 0 \leqslant t \leqslant x \\
\Psi(\lambda, 1+\alpha, t) \Phi_{1}(\lambda, 1+\alpha, x), \quad x \leqslant t
\end{array}\right.
$$

В частности, отсюда следует, что $R\left(\widetilde{A}_{\alpha, 0}-\lambda\right)$ плотно в $\Pi(\alpha)$, расширение $\widetilde{A}_{\alpha, 0}$ является самосопряженным в $\Pi(\alpha)$, а индексы дефекта оператора $A_{\alpha}$ равны 1 . Дефектное подпространство $\mathscr{N}_{\lambda}$ оператора $A_{\alpha}$ порождено функцией $\Psi(\lambda, 1+\alpha, x)$. Таким образом, $\operatorname{dim} A_{\alpha}^{+}\left(\bmod A_{\alpha}\right)=2$, а максимальное отношение $T_{\alpha}$ совпадает с $A_{\alpha}^{+}$и не зависит от выбора $\lambda \in \mathbb{C} \backslash \mathbb{R}$. Как отмечалось вьше, многозначная часть отношения $T_{\alpha}=A_{\alpha}^{+}$ порождена вектором $h=(0, \ldots, 0,1) \in \Pi(\alpha)$. 
Совокупность $\left\{\mathbb{C}, \Gamma_{0}, \Gamma_{1}\right\}$ образует граничную тройку отношения $T_{\alpha}=A_{\alpha}^{+}$в пространстве $\Pi(\alpha)$. Найдем соответствующую функцию Вейля оператора $A_{\alpha}$. Учитывая поведение функций $\Psi(\lambda, 1+\alpha, x), \Psi^{(n+1)}(\lambda, 1+\alpha, x)$ при $x \rightarrow 0$ :

$$
\begin{aligned}
\Psi(\lambda, 1+\alpha, x) & \asymp \frac{\Gamma(-\alpha)}{\Gamma(\lambda-\alpha)} \\
\Psi^{(n+1)}(\lambda, 1+\alpha, x) & =(-1)^{(n+1)}(\lambda)_{n+1} \Psi(\lambda+n+1, \alpha+n+2, x) \\
& \asymp(-1)^{(n+1)} \frac{\Gamma(\alpha+n+1)}{\Gamma(\lambda)} x^{-(\alpha+n+1)},
\end{aligned}
$$

получим из (20), (24)

$$
\Gamma_{0} \Psi(\lambda, 1+\alpha, x)=-\frac{\Gamma(\alpha+1)}{\Gamma(\lambda)}, \quad \Gamma_{1} \Psi(\lambda, 1+\alpha, x)=\frac{\Gamma(-\alpha)}{\Gamma(\lambda-\alpha)}
$$

Таким образом, функция Вейля $m_{\alpha}(\lambda)$ оператора $A_{\alpha}$, отвечающая граничной тройке $\left\{\mathbb{C}, \Gamma_{0}, \Gamma_{1}\right\}$, принимает вид

$$
m_{\alpha}(\lambda)=-\frac{\Gamma(-\alpha) \Gamma(\lambda)}{\Gamma(\lambda-\alpha) \Gamma(\alpha+1)}=-\frac{B(\lambda,-\alpha)}{\Gamma(\alpha+1)} .
$$

Принадлежность функции $m_{\alpha}(\lambda)$ обобщенному классу Неванлинны $N_{\varkappa}, \varkappa=[(n+1) / 2]$, видна непосредственно из разложения в ряд функции $m_{\alpha}(\lambda)($ см. $(9))$.

Подытожим результаты этого пункта.

Teоpema 2. Пусть $-n-1<\alpha<-n, n \in \mathbb{N}$. Tогдa

1) минимальный оператор Лагерра $A_{\alpha}$ является әрмитовым неплотно заданным оператором в пространстве $\Pi(\alpha)$ с индексами дефекта 1 ;

2) соотношения (20) определяют граничную тройку $\left\{\mathbb{C}, \Gamma_{0}, \Gamma_{1}\right\}$ отношения $A_{\alpha}^{+}$; соответствующая функиия Вейля $m_{\alpha}(\lambda)$ вычисляется по формуле (25) и принадлежит классу $N_{\varkappa}, \varkappa=[(n+1) / 2]$

3) всякое самосопряжснное расширение $\widetilde{A}_{\alpha, 0}$ оператора $A_{\alpha}$, отличное от $\widetilde{A}_{\alpha, \infty}=\operatorname{ker} \Gamma_{0}$, описывается граничнылм условием $\Gamma_{1} y=\theta \Gamma_{0} y, \theta \in \mathbb{R}$, его собственные числа совпадают с нулями функиии $m_{\alpha}(\lambda)-\theta$.

ЗАмЕчАниЕ 1. При стандартном определении минимального оператора как замыкания оператора $l_{\alpha}$, определенного первоначально на гладких финитных функциях, его индексы дефекта равны $\varkappa=[(n+1) / 2]$ (см. [3]). Мы намеренно избегаем этой ситуации, вводя иное определение минимального оператора $A_{\alpha}$.

5. Спектр. Рассмотрим самосопряженное расширение $\widetilde{A}_{\alpha, \infty}$, определяемое граничным условием $\Gamma_{0} y=0$. Спектр оператора $\widetilde{A}_{\alpha, \infty}$ состоит из простых собственных чисел $-j$, где $j \in \mathbb{Z}_{+}$, являющихся полюсами функции $m_{\alpha}(\lambda)$. Соответствующие собственные функции совпадают с полиномами Лагерра $L_{j}^{(\alpha)}(x), j \in \mathbb{Z}_{+}$. В силу соотношений

$$
\left\langle L_{j}^{(\alpha)}, L_{j}^{(\alpha)}\right\rangle_{\alpha}=\frac{\Gamma(j+\alpha+1)}{j !}, \quad j \in \mathbb{Z}_{+}
$$


оператор $\widetilde{A}_{\alpha, \infty}$ имеет лишь регулярные критические точки $n-1-2 j, j=0,1, \ldots$, $[(n-1) / 2]$ (см. [17]). Поэтому система собственных функций $\left\{L_{j}^{(\alpha)}\right\}_{j=0}^{\infty}$ полна в П $(\alpha)$ и для всякого элемента $f \in \Pi(\alpha)$ справедливо разложение

$$
f=\sum_{j=0}^{\infty} \frac{\left\langle f, L_{j}^{(\alpha)}\right\rangle_{\alpha}}{\Gamma(\alpha+j+1)} j ! L_{j}^{(\alpha)}
$$

сходящееся в $\Pi(\alpha)$. Стандартные рассуждения (см. [18]) показьвают, что ряд (26) равномерно сходится на компактах $[c, d](0<c<d)$ для всех функций $f(x) \in C^{\infty}\left(\mathbb{R}_{+}\right) \cap \Pi(\alpha)$.

Самосопряженное расширение $\widetilde{A}_{\alpha, 0}$, определяемое граничным условием $\Gamma_{1} y=0$, является линейньм отношением с многозначной частью mul $\widetilde{A}_{\alpha, 0}$, порожденной вектором $h=(0, \ldots, 0,1) \in \Pi(\alpha)$. Заметим, что вектор $h$ допускает следующее разложение в ряд:

$$
h=\frac{1}{\Gamma(\alpha+1)} \sum_{j=0}^{\infty} L_{j}^{(\alpha)},
$$

который сходится в П $(\alpha)$. Спектр отношения $\widetilde{A}_{\alpha, 0}$ состоит из простых собственных чисел $\alpha-j$, где $j \in \mathbb{Z}_{+}$. Соответствующие собственные функции имеют вид $x^{-\alpha} L_{j}^{(-\alpha)}(x)$, $j \in \mathbb{Z}_{+}$. Функция Вейля $-1 / m_{\alpha}(\lambda)$, соответствуюшая расширению $\widetilde{A}_{\alpha, 0}$, растет как $\lambda^{-\alpha}$ при $\lambda \rightarrow \infty$. Поэтому $\infty$ является сингулярной критической точкой отношения $\widetilde{A}_{\alpha, 0}$ (см. [17]).

Проследим за динамикой собственных чисел расширения $\widetilde{A}_{\alpha, \theta}$ при изменении параметра $\theta$ в интервале $(-\infty, \infty)$. Например, при $\alpha=-5 / 2$ функция Вейля $m_{\alpha}(\lambda)$ имеет вид, указанный на рис. 1.

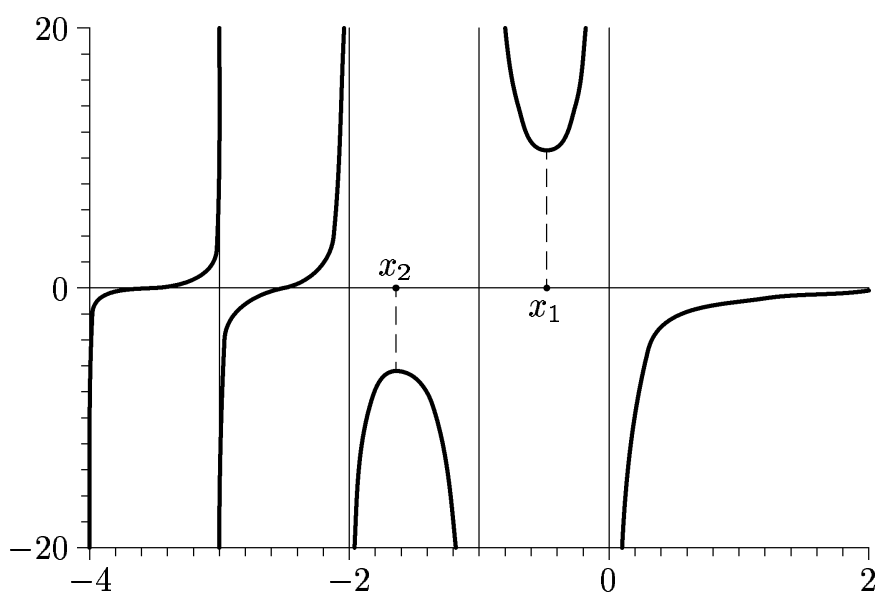

Рис. 1

Положим

$$
\theta_{1}=\min _{(-1,0)} m_{\alpha}(x)=m_{\alpha}\left(x_{1}\right), \quad \theta_{2}=\max _{(-2,-1)} m_{\alpha}(x)=m_{\alpha}\left(x_{2}\right)
$$


Собственные числа $\lambda_{j}(\theta)$ расширения $\widetilde{A}_{\alpha, \theta}$ совпадают в силу теоремы 2 с нулями функции $m_{\alpha}(\lambda)-\theta$. Из рис. 1 видно, что $\lambda_{j}(\theta)$ являются монотонно возрастающими функциями от $\theta$ со значениями в $(-j,-j+1)$ при $j \geqslant 3$, а $\lambda_{0}(\theta)$ - монотонно возрастающей функцией от $\theta \in(-\infty, 0)$ со значениями в $(0, \infty)$. В то же время поведение собственных чисел $\lambda_{1}(\theta), \lambda_{2}(\theta)$ является несколько необычным. При возрастании $\theta$ в интервале $\left(-\infty, \theta_{1}\right)$ собственные числа $\lambda_{1}(\theta), \lambda_{2}(\theta)$, находясь в интервале $(-2,-1)$, двигаются навстречу друг другу. После слияния при $\theta=\theta_{1}$ они отрьваются от вещественной оси в точке $x_{1}$ и, описьвая симметричные дуги в $\mathbb{C}_{+}$и $\mathbb{C}_{-}$, опускаются на интервал $(-1,0)$ в точке $x_{0}$ при $\theta=\theta_{0}$. При дальнейшем возрастании $\theta$ собственные числа $\lambda_{1}(\theta)$ и $\lambda_{2}(\theta)$ двигаются к концам интервала $(-1,0)$. Такое поведение собственных чисел является характерным для эрмитовых операторов в пространстве Понтрягина и в общем случае описано в [14].

6. Энергетическая форма. Будем говорить, что эрмитов оператор $A$ в пространстве Понтрягина $\left(\Pi_{\varkappa},[\cdot, \cdot]\right)$ обладает $N^{k}$-свойством, если форма $[A f, f], f \in \mathscr{D}(A)$, имеет $k$ отрицательных квадратов. Как показано в [19], о наличии у оператора $A$ $N^{k}$-свойства можно судить по поведению его функции Вейля. Так, если $A-$ простой эрмитов оператор в пространстве Понтрягина с индексами дефекта 1 , a $m(\lambda)$ - его функция Вейля, имеющая на полуоси $(-\infty, 0)$ лиш конечное число особых точек, и такая, что существует предел

$$
m(-\infty)=\lim _{x \downarrow-\infty} m(x),
$$

то справедлива эквивалентность

$$
A \text { обладает } N^{k} \text {-свойством } \Longleftrightarrow \lambda(m(\lambda)-m(-\infty)) \in N_{k} \text {. }
$$

Условие (27) может быть переписано в виде $m(\lambda)-m(-\infty) \in N_{\varkappa}^{k}$, если использовать обозначение $N_{\varkappa}^{k}$ для класса функций $m(\lambda) \in N_{\varkappa}$ таких, что $\lambda m(\lambda) \in N_{k}$ (см. [19]).

Если к тому же функция Вейля $m(\lambda)$ оператора $A$ удовлетворяет условию

$$
\lim _{x \uparrow 0} m(x)=\infty
$$

то описание всех его расширений $\widetilde{A}_{\alpha, \theta}$, обладающих $N^{k}$-свойством, имеет вид (см. [19])

$$
A \text { обладает } N^{k} \text {-свойством } \Longleftrightarrow \theta-m(-\infty) \leqslant 0 \text {. }
$$

Применяя эквивалентности $(27),(28)$ к оператору $-A_{\alpha}$, получим

ПРЕДЛОЖЕНИЕ 2. Пусть $-n-1<\alpha<-n, n \in \mathbb{N}, A_{\alpha}-$ минимальный оператор Лагерра, $m_{\alpha}(\lambda)$ - его функиия Вейля вида (25). Тогда

1) функиия $m(\lambda)=-m_{\alpha}(-\lambda)$ принадлежит классу $N_{\varkappa}^{k}$, где $\varkappa=[(n+1) / 2]$, $k=[n / 2]$

2) $-\widetilde{\sim}_{\alpha}$ обладает $N^{k}$-свойством;

3) $-\widetilde{A}_{\alpha, \theta}$ обладает $N^{k}$-свойством, если и только если $\theta \geqslant 0$ или $\theta=\infty$, в противном случае $-A_{\alpha}$ обладает $N^{k+1}$-свойством. 
ДокАЗАТЕЛЬСТво. Функция $\lambda m(\lambda)=-\lambda m_{\alpha}(-\lambda)$ допускает разложение в ряд

$$
\lambda m(\lambda)=\frac{1}{\Gamma(\alpha+1)} \sum_{j=0}^{\infty} \frac{(\alpha+1)_{j}}{j !}\left(\frac{j}{j-\lambda}-1\right),
$$

из которого следует, что $\lambda m(\lambda) \in N_{k}, k=[n / 2]$. Таким образом, $m(\lambda) \in N_{\varkappa}^{k}$.

Так как функция $m(\lambda)=-m_{\alpha}(-\lambda)$ является функцией Вейля оператора $-A_{\alpha}$, справедливость утверждений 2), 3) следует из эквивалентностей (27), (28).

Отметим, что расширения $-\widetilde{A}_{\alpha, 0},-\widetilde{A}_{\alpha, \infty}$ являются фридрихсовским и крейновским расширениями оператора $-A_{\alpha}$ (в терминологии работы [19]).

\section{СПИСОК ЦИТИРОВАННОЙ ЛИТЕРАТУРЫ}

[1] Morton R. D., Krall A. M. Distributional weight functions for orthogonal polynomials // SIAM J. Math. Anal. 1978. V. 2. P. 604-626.

[2] Krall A. M. Laguerre polynomial expansions in indefinite inner product spaces // J. Math. Anal. Appl. 1979. V. 70. P. 267-279.

[3] Krall A. M. On boundary values for the Laguerre operator in indefinite inner product spaces // J. Math. Anal. Appl. 1982. V. 85. P. 406-408.

[4] Mingarelli A. B., Krall A. M. Jacobi-type polynomials under an indefinite inner product // Proc. Roy. Soc. Edinburgh. Sect. A. 1981. V. 90. P. 147-153.

[5] Березин Ф. А. О модели Ли // Матем. сб. 1963. Т. 60 (120). № 4. С. 425-446.

[6] Шондин Ю. Г. Обобщенные точечные взаимодействия в $\mathbb{R}^{3}$ и связанные с ними модели // ТMФ. 1985. T. 65. №1. C. 24-34.

[7] Jonas P., Langer H., Textorius B. Models and unitary equivalence of cyclic selfadjoint operators in Pontrjagin spaces // Oper. Theory Adv. Appl. 1992. V. 59. P. 252-284.

[8] Азизов Т.Я., Иохвидов И. С. Основы теории линейных операторов в пространствах с индефинитной метрикой. М.: Наука, 1986.

[9] Dijksma A., de Snoo H.S. V. Symmetric and selfadjoint relations in Krein spaces. I // Oper. Theory Adv. Appl. 1987. V. 24. P. 145-166.

[10] Маламуд М. М. Об одной формуле для обобщенных резольвент неплотно заданного эрмитова оператора // Укр. матем. ж. 1992. Т. 44. № 12. С. 1658-1688.

[11] Деркач В.А. О расширениях неплотно заданного эрмитова оператора в пространстве Крейна // Докл. АН УССР. Сер. А. 1990. № 10. С. 15-19.

[12] Derkach V.A., Malamud M. M. The extension theory of Hermitian operators and the moment problem // J. Math. Sci. 1995. V. 73. № 2. P. 141-242.

[13] Горбачук В. И., Горбачук М. Л. Граничные задачи для дифференциально-операторных уравнений. Киев: Наукова думка, 1984.

[14] Крейн М. Г., Лангер Г. К. О дефектных подпространствах и обобщенных резольвентах эрмитова оператора в пространстве $\Pi_{\varkappa} / /$ Функцион. анализ и его прилож. 1971. Т. 5. № 2. C. $59-71$.

[15] Abramowitz M., Stegun I. Handbook of Mathematical Functions with Formulas, Graphs and Mathematical Tables. Washington: Department of Commerce, 1964.

[16] Уиттекер Е. Т., Ватсон Г. Н. Курс современного анализа. Т. 2. М.: Физматгиз, 1963.

[17] Крейн М. Г., Лангер Г. К. О спектральной функции самосопряженного оператора в пространстве с индефинитной метрикой // Докл. АН СССР. 1963. Т. 152. №1. С. 39-42.

[18] Суетин П. К. Классические ортогональные многочлены. М.: Наука, 1976.

[19] Derkach V. On Weyl function and generalized resolvents of a Hermitian operator in a Krein space // Integral Equations Operator Theory. 1995. V. 23. P. 387-415. 